Perez-Lasierra, J.L.; Hernández-Vicente, A.; Comeras-Chueca, C.; Casajús-Mallén, J.A. (2021). Are Tomorrow's Spanish Doctors Prepared to Combat Physical Inactivity? Revista Internacional de Medicina y Ciencias de la Actividad Física y el Deporte vol. 21 (83) pp. 467-477. Http://cdeporte.rediris.es/revista/revista83/artestan1261.htm

DOI: https://doi.org/10.15366/rimcafd2021.83.004

\title{
ORIGINAL
}

\section{¿ESTÁN PREPARADOS LOS MÉDICOS ESPAÑOLES DEL MAÑANA PARA COMBATIR LA INACTIVIDAD FÍSICA?}

\section{ARE TOMORROW'S SPANISH DOCTORS PREPARED TO COMBAT PHYSICAL INACTIVITY?}

\author{
Perez-Lasierra, J.L.'; Hernández-Vicente, A. ${ }^{1}$; Comeras-Chueca, C. $^{1}$ y \\ Casajús-Mallén, J.A. ${ }^{2}$
}

${ }^{1}$ Estudiantes de doctorado de la Universidad de Zaragoza, Facultad de Ciencias de la Salud, Grupo de investigación GENUD, Zaragoza (España) ilperez@unizar.es, ahernandez@unizar.es, ccomeras@unizar.es

${ }^{2}$ Catedrático Universidad de la Universidad de Zaragoza, Facultad de Ciencias de la Salud, Grupo de investigación GENUD, Zaragoza,(España) joseant@unizar.es

Financiación: JLPL y AHV son beneficiarios de subvenciones FPU 2016 del Ministerio de Educación Cultura y Deporte (España); y CCC es beneficiaria desde 2017 de una subvención destinada a la contratación de personal investigador predoctoral en formación de la Diputación General de Aragón.

Agradecimientos: Los autores quieren dar las gracias por su colaboración a todos los participantes y al Comité Organizador del XI Congreso de Educación Médica. Igualmente merecen un especial agradecimiento todos los integrantes del grupo de investigación GENUD por su participación en la toma de datos.

Código UNESCO I UNESCO Code: 5899 Otras especialidades Pedagógicas (Actividad Física y Salud) / Other Educational Specialties (Physical Activity and Health)

Clasificación Consejo de Europa / Clasificación del Consejo de Europa I Council of Europe Classification: 5. Didáctica y metodología / Didactics and methodology

Recibido 24 junio de 2019 Received June 24, 2019

Aceptado 30 de noviembre de 2019 Accepted November 30, 2019

\section{RESUMEN}

El objetivo del estudio fue averiguar que conocimientos tenían los estudiantes de medicina españoles sobre las recomendaciones actuales de Actividad Física (AF) de la Organización Mundial de la Salud (OMS). Un total de 334 estudiantes 
de medicina $\left(2^{\circ}-6^{\circ}\right.$ curso) de 29 universidades españolas cumplimentaron un cuestionario de conocimientos sobre las recomendaciones de AF de la OMS. EI $100 \%$ de los encuestados afirmaron que recomendar AF es una parte importante del trabajo de un médico, pero sólo un $34,1 \%$ se sentían preparados para ello. Un $85,0 \%$ y un $72,5 \%$ desconocían las recomendaciones de AF de la OMS para adultos y niños respectivamente. El 94,3\% demandaron una mayor formación académica en AF, siendo el método de preferencia la asignatura optativa. La falta de conocimiento sobre las recomendaciones de AF de los estudiantes de medicina españoles es evidente y alarmante. Aumentar la formación en dichos contenidos mediante asignaturas optativas podría revertir esta situación.

PALABRAS CLAVE: Ejercicio; Prestación de asistencia sanitaria; Enfermedades no transmisibles; Currículum; Educación médica.

\section{ABSTRACT}

The aim of this study was to discover the level of knowledge that Spanish medical students had about the current global physical activity (PA) recommendations provided by the World Health Organization (WHO). A total of 334 medical students $\left(2^{\text {nd }}-6^{\text {th }}\right.$ degree years) from 29 Spanish universities completed a questionnaire of knowledge about WHO's global PA recommendations. All respondents $(100 \%)$ stated that recommending PA is an important part of a physician's role, but only $34.1 \%$ felt prepared for it. $85.0 \%$ and $72.5 \%$ of respondents did not know the PA recommendations for adults and children respectively. Likewise, $94.3 \%$ of respondents demanded more education in PA, with the elective course being the preferred method of learning. The lack of knowledge regarding PA recommendations by medical students is evident and alarming. Increasing the education focused on these contents through elective courses could reverse this situation.

KEY WORDS: Exercise; Delivery of Health Care; Noncommunicable Diseases; Curriculum; Medical Education.

\section{INTRODUCCIÓN}

La inactividad física es la cuarta causa de mortalidad en los países desarrollados (1). A pesar de la eficacia demostrada por la Actividad Física (AF) para prevenir las enfermedades con mayor tasa de mortalidad de la actualidad, el 66,8\% de los españoles no alcanza los niveles de AF recomendados por la Organización Mundial de la Salud (OMS) (2). Aunque la esperanza de vida en los países desarrollados sea cada vez más alta, no se corresponde con la "esperanza de vida saludable", definida como el número de años que se puede esperar que una persona viva con completa salud (3). En España, esta diferencia es cada vez mayor, en 2016 el porcentaje de vida saludable respecto al total era de 53,7\% para los hombres y $44,0 \%$ para las mujeres (4), lo que se ve acompañado por un descenso en la calidad de vida, asociado principalmente a la pérdida de capacidad funcional (5). La relación entre inactividad física y perdida de capacidad funcional es estrecha y directa (6). 
Las enfermedades cardiovasculares (ECV) son la causa mas frecuente de mortalidad en las sociedades desarrolladas (7). Las ECV se asocian con la existencia de una serie de factores de riesgo previos que desencadenan finalmente la patología. Los factores de riesgo más habituales como la inactividad física, una inadecuada alimentación, tabaquismo, hipercolesterolemia, hipertensión arterial, diabetes u obesidad, están asociados a un estilo de vida poco saludable (8). Diversas investigaciones han demostrado que si elimináramos la inactividad física, ya catalogada como pandemia de primera prioridad (9), se reduciría: la mortalidad general, el cáncer de colon y mama, la diabetes tipo II y otras muchas patologías (hasta 26) (10), lo que se traduciría en un aumento de la esperanza de vida de 0,68 años a nivel mundial, y 0,78 años en España $(11,12)$, y también en una mejor calidad de vida durante estos años adicionales $(13,14)$. La AF ha demostrado ser un factor protector contra las enfermedades no transmisibles y sus factores de riesgo de tanta 0 incluso mayor eficacia que algunos medicamentos, llegando a denominarse la verdadera poli-píldora (15).

Si se valora el número de European Credit Transfer and Acumulation System (ECTS) y contenidos en AF y salud del grado de medicina de diferentes universidades españolas, se puede observar que sólo el $2,38 \%$ del total se destinan a contenidos relacionados con la actividad y/o el ejercicio físico como herramientas de salud (16). Las mismas carencias formativas se evidencian en países como EEUU (17), Australia (18), Turquía (19) o Inglaterra (20). Recientes estudios llevados a cabo en Reino Unido han manifestado la falta de conocimientos por parte de los estudiantes de medicina sobre las guías mundiales de recomendaciones de AF para la salud $(21,22)$.

Por lo tanto, el objetivo del presente estudio fue evaluar los conocimientos que tienen sobre las guías mundiales de recomendaciones de AF para la salud los estudiantes de medicina de las universidades españolas.

\section{MÉTODOS}

En el estudio participaron 334 de los 700 estudiantes de medicina que asistieron al XI Congreso de Educación Médica que tuvo lugar en Zaragoza en 2018. Los participantes eran de varios cursos del grado $\left(2^{\circ}-6^{\circ}\right)$ y provenían de 29 universidades españolas diferentes, tanto públicas como privadas, sus características se muestran en la tabla 1 . Los alumnos de $1^{\text {er }}$ curso no han sido considerados para el estudio dado su reducido bagaje de conocimientos y su poca experiencia en el grado de medicina. 
Tabla 1. Número de estudiantes por universidad y curso.

CURSO

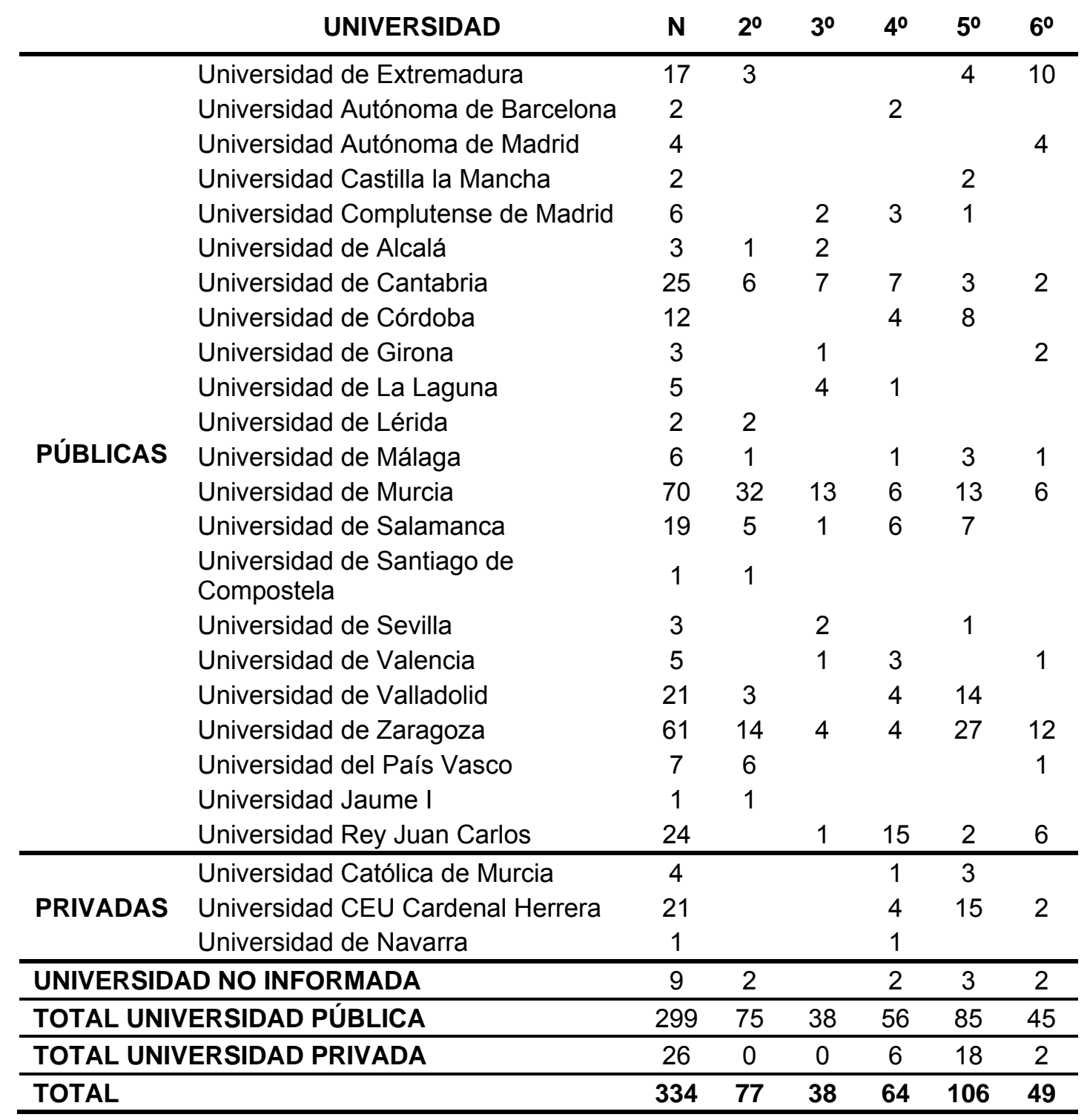

A todos los participantes se les pidió que cumplimentaran un cuestionario de conocimientos sobre las recomendaciones actuales de AF para la salud de la OMS; dicho cuestionario es el utilizado por Osborne et al. en la universidad de Edimburgo. El cuestionario utilizado se puede consultar en el suplemento 1 (Physical Activity For Health Questionnaire).

El análisis estadístico se basó en realizar frecuencias de las respuestas del cuestionario para todos los participantes, y segmentando los resultados de las respuestas en función del curso en el que se encontraban. También se realizaron T-test para comprobar si existían diferencias de conocimientos entre grupos basados en la autopercepción de preparación para aconsejar AF. 


\section{RESULTADOS}

\section{PARA TODA LA MUESTRA:}

De los 334 estudiantes que cumplimentaron el cuestionario, todos estaban muy de acuerdo $(83,8 \%)$ o de acuerdo $(16,2 \%)$ en que aconsejar a los pacientes sobre AF es una parte importante de las funciones del médico, y además un $34,1 \%$ afirmaron sentirse bien preparados para aconsejar AF a la población general, mientras que un $65,9 \%$ no se sentían suficientemente preparados para ello.

Por otro lado, un $94,3 \%$ de los 334 participantes manifestaron que les gustaría recibir una mayor preparación académica sobre AF, incluyendo contenidos sobre epidemiología, beneficios para la salud, promoción, indicaciones y prescripción de ejercicio físico. Cuando se les preguntó de qué modo les gustaría recibir mayor preparación académica sobre AF un 16,8\% eligieron la opción de materia obligatoria, un $53,5 \%$ la de materia optativa, un $42,6 \%$ la de actividad académica complementaria, un $34,8 \%$ la de contenido teórico transversal, un $35,1 \%$ la de casos clínicos en otras asignaturas, un $18,9 \%$ la de curso presencial fuera de la universidad y un $16,8 \%$ la de curso informático (figura 1).

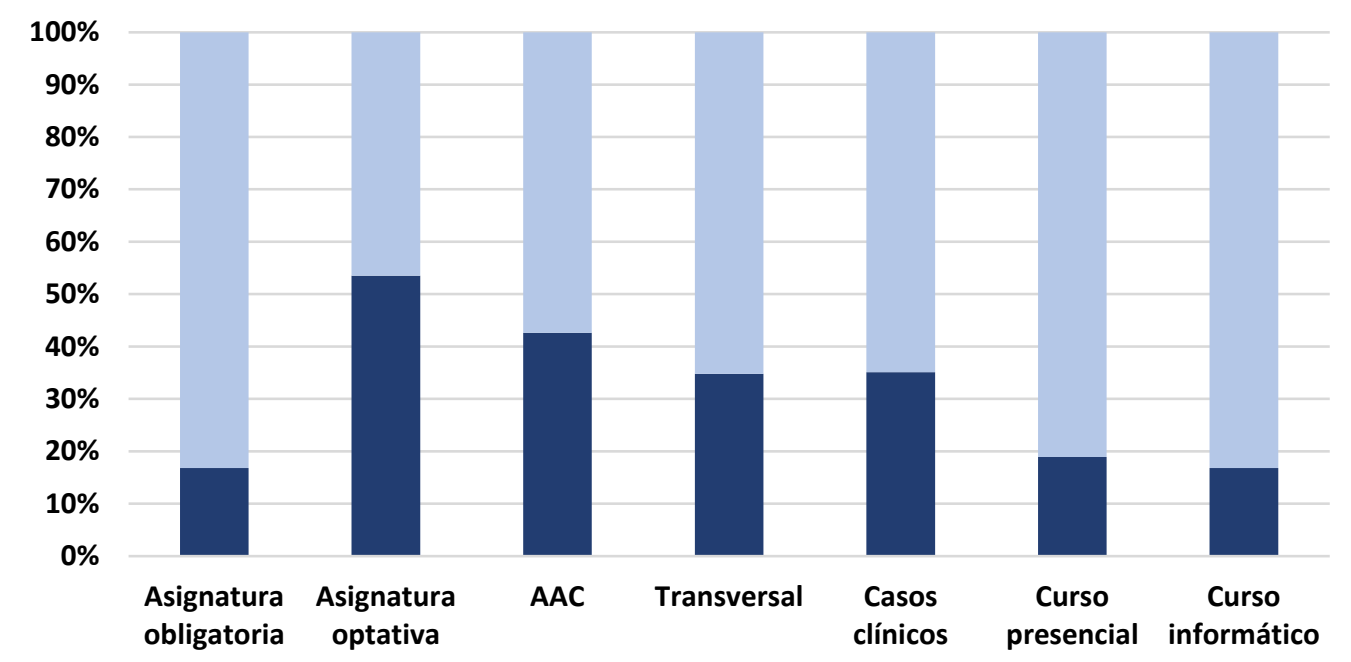

Figura 1. Método de enseñanza demandado por los estudiantes de medicina españoles para recibir una mayor preparación en contenidos relacionados con la actividad física para la salud.

Abreviaturas: $A A C=$ Actividad académica complementaria sobre prescripción de ejercicio;

Transversal= Contenido teórico transversal en otras asignaturas; Casos clínicos= Casos

clínicos en otras asignaturas; Curso presencial= Curso presencial fuera de la Universidad.

En relación a los conocimientos que poseían sobre las recomendaciones de AF se obtuvieron los siguientes resultados: a la pregunta de "cuántos minutos de actividad física moderada (AFM) deben realizar los adultos en una semana como mínimo", un $85,0 \%$ dieron un valor erróneo (infraestimando o sobrestimando el valor), un $2,1 \%$ no respondieron, y un $12,9 \%$ de los participantes dieron una respuesta correcta (figura 2). A la pregunta de "cuántos minutos de actividad física moderada a vigorosa (AFMV) deben acumular los niños y jóvenes al día como mínimo", un $72,5 \%$ dieron una respuesta errónea (infraestimando o 
sobreestimando el valor), un 3,6\% no respondieron, y un $24,0 \%$ respondieron de manera correcta (figura 3). Un $65,9 \%$ de los participantes respondieron erróneamente a las dos preguntas anteriores, un $31,4 \%$ acertó una de las dos preguntas, y un $2,7 \%$ respondieron correctamente a ambas preguntas.

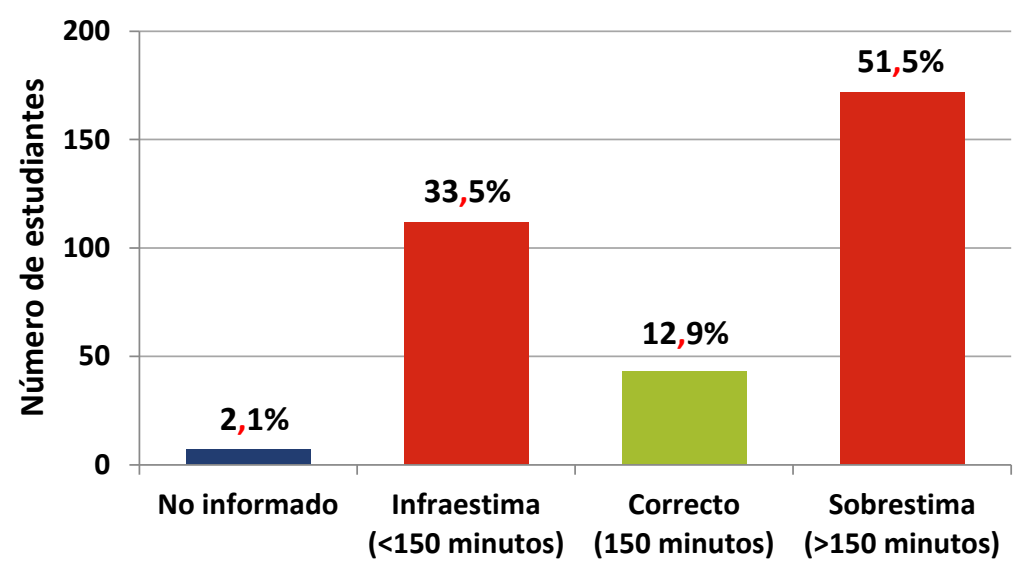

Figura 2. Frecuencias de respuestas a la pregunta: ¿Cuántos minutos de actividad física moderada deben acumular los adultos como mínimo en una semana?

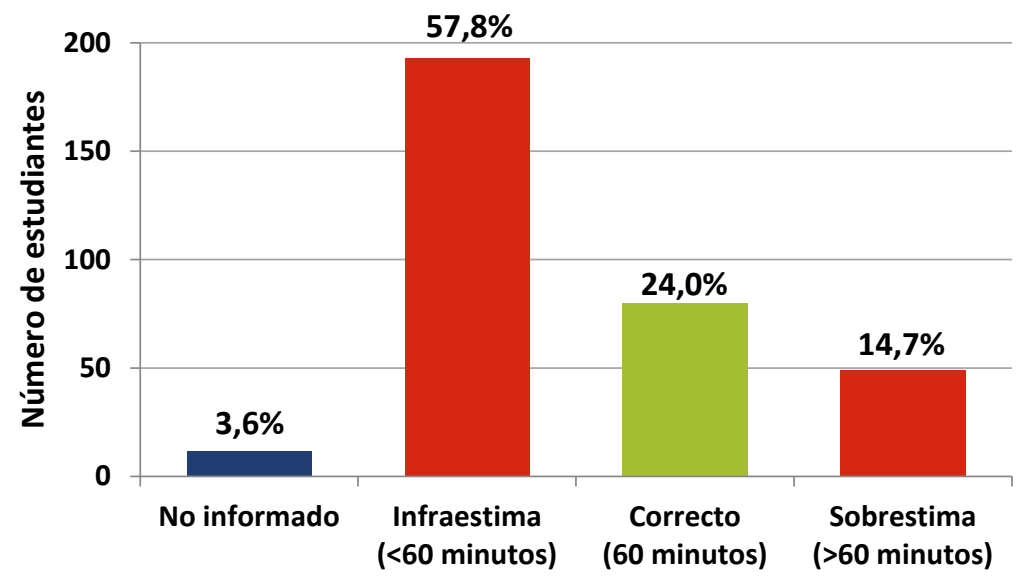

Figura 3. Frecuencia de respuestas a la pregunta: ¿Cuántos minutos al día de actividad física moderada-vigorosa deben acumular como mínimo los niños y jóvenes?

En la figura 4 se puede observar como al comparar los conocimientos de las recomendaciones de AF de la OMS para los diferentes grupos de edad, en base a la autopercepción de preparación para dar un buen consejo sobre AF, no existen diferencias significativas entre los conocimientos demostrados $(p>0,05)$. 


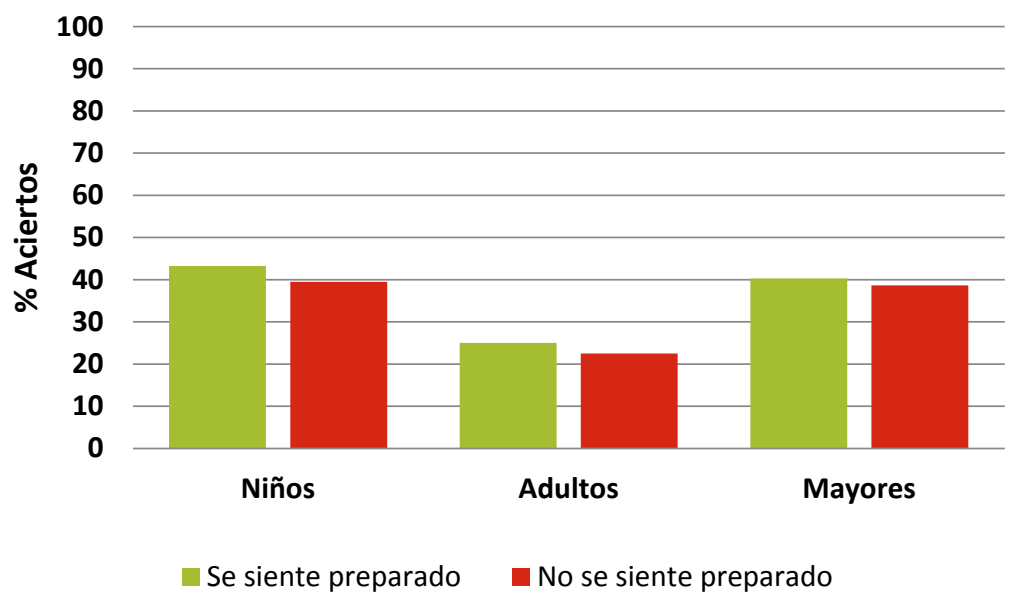

Figura 4. Porcentaje de aciertos sobre las recomendaciones mundiales de actividad física para cada grupo de edad en base a la autopercepción de preparación de los participantes para dar un buen consejo sobre actividad física.

\section{PARA ALUMNOS DE ÚLTIMOS CURSOS}

De los 334 alumnos que cumplimentaron el cuestionario, 219 eran alumnos de $4^{\circ}, 5^{\circ}$ o $6^{\circ}$ curso. Todos estaban muy de acuerdo $(87,7 \%)$ o de acuerdo $(12,3 \%)$ en que aconsejar a los pacientes sobre AF es una parte importante de las funciones del médico, y además un 37,3\% afirmaron sentirse bien preparados para aconsejar AF a la población general, mientras que un $62,7 \%$ no se sentían suficientemente preparados para ello. Cuando se les preguntó: "cuántos minutos de AFM deben realizar los adultos en una semana como mínimo", un $81,7 \%$ dieron un valor erróneo (infraestimando o sobrestimando el valor), un $1,4 \%$ no respondieron, y un $16,9 \%$ de los participantes dieron un respuesta correcta. A la pregunta de: "cuántos minutos de AFMV deben acumular los niños y jóvenes al día como mínimo", un 70,8\% dieron una respuesta errónea (infraestimando o sobreestimando el valor), un $3,2 \%$ no respondieron, y un $26,0 \%$ respondieron de manera correcta.

\section{DISCUSIÓN}

El éxito de un buen tratamiento se sustenta en un correcto diagnóstico; si no se es capaz de identificar a los sujetos inactivos, difícilmente se podrá actuar sobre esta anomalía conductual.

Todos los estudiantes de medicina están de acuerdo en que aconsejar a los pacientes AF es una parte importante de su trabajo. Sin embargo, pese a que aproximadamente un tercio de los encuestados afirma sentirse bien preparado para recomendar AF a la población general, la mayoría $(97,3 \%)$ no conoce las recomendaciones mundiales sobre AF para la salud de la OMS. El presente artículo pone de manifiesto que, para los estudiantes de medicina, los contenidos curriculares relacionados con la AF y la salud de los planes de estudio del grado de medicina son insuficientes; los estudiantes españoles demandan una mayor preparación académica en dicho campo (94,3\% de los encuestados), siendo la materia optativa el método de enseñanza más demandado. 
Este hándicap no solo tiene lugar en España, también se observa la misma tendencia a nivel mundial. Si se comparan estos resultados con los obtenidos por Osborne et al. en la Universidad de Edimburgo, se puede observar como los conocimientos demostrados por los estudiantes sobre las recomendaciones de AF para la población adulta son similares, ya que un $85,1 \%$ vs. $85,0 \%$ respondieron de manera errónea, de igual manera, si atendemos a las recomendaciones para los niños, un $75,7 \%$ vs. $72,5 \%$ no dieron una solución correcta (22). En la misma línea, otro estudio llevado a cabo en Escocia también pone de manifiesto el desconocimiento de las recomendaciones de AF para la población general entre los estudiantes de medicina de dos universidades (21).

Esta carencia de conocimiento mostrada por los estudiantes de medicina $(21,22)$, se encuentra presente en el sistema sanitario actual, ya que como refiere Wheeler et al., los médicos, enfermeros y demás personal sanitario tampoco conocen las recomendaciones de AF para la población general ni los beneficios que supone llevar una vida activa (23). Llegados a este punto deberíamos preguntarnos si el sistema sanitario está cumpliendo con la "Lex artis ad hoc", dado que a pesar de las irrefutables evidencias científicas a favor de la AF (15), la "praxis sanitaria" al respecto parece ser claramente mejorable. Se entiende que el graduado en medicina no debe ser experto en prescripción de ejercicio físico, pero sí debe tener unos conocimientos sobre AF que le permitan dar un buen consejo a sus pacientes (24), cumpliendo con la "Lex artis ad hoc". Si se aspira a un sistema sanitario más eficaz y sostenible, se debería plantear la inclusión de las recomendaciones de AF y la prescripción de ejercicio físico dentro de la cartera de servicios sanitarios.

A nivel mundial, los planes de estudio del grado de medicina apenas incluyen contenidos relacionados con la AF y su papel dentro de la salud, y los que lo incluyen lo hacen de manera deficiente (16-20). Este es un problema que se debe solucionar aportando nuevos conocimientos sobre AF y salud no solo a los estudiantes de medicina, sino a todos los estudiantes de las profesiones sanitarias que próximamente pasarán a formar parte del sistema sanitario (25). La preferencia de los estudiantes para recibir una mayor formación en conocimientos sobre AF y salud es diferente entre los estudios, unos prefieren una formación tipo taller (workshop) (22), y otros como en el presente estudio demandan una asignatura optativa. A pesar de esas pequeñas discrepancias, lo que parece evidente es que aumentar el número de ECTS y los contenidos del grado y postgrado relacionados con la prevención y tratamiento de enfermedades crónicas a través de la actividad y el ejercicio físico, favorecería una mayor promoción de la AF para la salud por parte de los médicos y personal sanitario, provocando un aumento en los niveles de AF en la población que se vería reflejado en el aumento de salud y calidad de vida de la población y disminución del gasto sanitario $(25,26)$. Un reciente estudio llevado a cabo en Turquía pone de manifiesto como una mayor formación sobre AF y salud en estudiantes de medicina puede ayudar a que los futuros médicos crean en el papel de la actividad y ejercicio físico dentro de la salud, y se sientan más capaces de dar un buen consejo sobre AF a sus pacientes (19). 
La principal fortaleza del estudio es que se trata de la primera investigación que analiza los conocimientos sobre las recomendaciones de AF mundiales que tienen los estudiantes de medicina españoles, contando además con datos de 29 universidades de diferente tipología (públicas y privadas) y procedencia geográfica. El tamaño muestral y su heterogeneidad son elementos que se deben de tener en cuenta en la interpretación de los resultados.

\section{CONCLUSIONES}

En función de los resultados obtenidos se puede concluir que los estudiantes de medicina evaluados desconocen, en su mayoría, las recomendaciones mundiales de AF de la OMS, y que deberían mejorar su formación en esta materia mediante asignaturas optativas o complementos de formación.

\section{REFERENCIAS BIBLIOGRÁFICAS}

1. WHO. Recomendaciones mundiales sobre actividad física para la salud [Internet]. Suiza; 2010. Available from: https://apps.who.int/iris/bitstream/handle/10665/44441/9789243599977_ spa.pdf;jsessionid=CE6D3919D9B8BE35F870993174FB5AD2?sequenc $\mathrm{e}=1$

2. Fernandez-navarro $P$, Aragones $T$, Ley V. Leisure-time physical activity and prevalence of non-communicable pathologies and prescription medication in Spain. PLoS One. 2018;19:1-13. https://doi.org/10.1371/journal.pone.0191542

3. WHO. WHO: World Health Statistics 2009 [Internet]. 2009. Available from: https://www.who.int/gho/publications/world_health_statistics/EN_WHS09 Full.pdf

4. $\quad$ Abellan A, Aceituno P, Pérez J, Ramiro D, Ayala A, Pujol R. Informes envejecimiento en red. Un perfil de las personas mayores en España, 2019. Vol. 22. 2019.

5. López-garcía E, Banegas JR, Pérez-regadera AG. Valores de referencia de la versión española del Cuestionario de Salud SF-36 en población adulta de más de 60 años. Med Clin. 2003;120(15):568-73.

https://doi.org/10.1016/S0025-7753(03)73775-0

6. Harridge S, Lazarus NR. Physical Activity , Aging , and Physiological Function. Physiology. 2017;32:152-61.

https://doi.org/10.1152/physiol.00029.2016

7. Collaborators G 2016 C of D. Global, regional, and national age-sex specific mortality for 264 causes of death , $1980-2016$ : a systematic analysis for the Global Burden of Disease Study 2016. Lancet. 2017;390:1980-2016.

8. Joseph P, Leong D, Mckee M, Anand SS, Schwalm J, Teo K, et al. Reducing the Global Burden of Cardiovascular Disease, Part 1. Circ Res. 2017;677-95. https://doi.org/10.1161/CIRCRESAHA.117.308903

9. Kohl HW, Craig CL, Lambert EV, Inoue S, Alkandari JR, Leetongin G, et al. The pandemic of physical inactivity: global action for public health. Lancet. Elsevier Ltd; 2012;380(9838):294-305.

https://doi.org/10.1016/S0140-6736(12)60898-8 
10. Pedersen BK, Saltin B. Exercise as medicine - evidence for prescribing exercise as therapy in 26 different chronic diseases. Scand J Med Sci Sports. 2015;25(Suppl. 3):1-72. https://doi.org/10.1111/sms.12581

11. Lee M, Shiroma E, Lobelo F, Puska P. Impact of Physical Inactivity on the World's Major Non-Communicable Diseases. Lancet. 2012;380(9838):219-29. https://doi.org/10.1016/S0140-6736(12)61031-9

12. Lear SA, Hu W, Rangarajan S, Gasevic D, Leong D, lqbal R, et al. The effect of physical activity on mortality and cardiovascular disease in 130 000 people from 17 high-income, middle-income, and low-income countries : the PURE study. Lancet. 2017;390(10113):2643-54. https://doi.org/10.1016/S0140-6736(17)31634-3

13. Jürgens I. Práctica deportiva y percepciónde calidad de vida / Sport practice and perception of quality of life. Rev Int Med y Ciencias la Act Física y el Deport. 2006;6(22):66-74.

14. Del Valle Soto M, Prieto Saborit JA, Nistal Hernández P, Martínez Suárez PC, Ruíz Fernández L. Impacto de las estrategias de ejercicio físico en la CVRS de adultos sedentarios / Impact Exercise Strategies in the HRQOL of the Sedentary Adults. Rev Int Med y Ciencias la Act Física y del Deport. 2016;64(2016):739-56.

https://doi.org/10.15366/rimcafd2016.64.008

15. Fiuza-luces C, Garatachea N, Berger NA, Lucia A. Exercise is the Real Polypill. Physiology. 2013;28:330-58.

https://doi.org/10.1152/physiol.00019.2013

16. Calonge S, Casajús JA, Gonzalez-Gross M. La actividad física en el currículo español de los grados de Medicina y Ciencias de la Actividad Fisica y del Deporte como recurso en la prevención y tratamiento de las enfermedades crónicas. Nutr Hosp. 2017;34(4):961-8. https://doi.org/10.20960/nh.730

17. Cardinal $B$, Park E, Kim M, Cardinal M. If exercise is medicine, where is exercise in medicine? Review of U.S. medical education curricula for physical activity-related content. J Phys Act Health. 2015;12:1336-43. https://doi.org/10.1123/jpah.2014-0316

18. Strong A, Stoutenberg M, Hobson-Powell A, Hargreaves M. An evaluation of physical activity training in Australian medical school curricula. J Sci Med Sport. Sports Medicine Australia; 2016.

19. Dönmez G, Torğutalp Ş, Yargıç MP, Karanfil Y. The Effects of Elective Sports Medicine Internship on Physical Activity Counselling Attitude of Medical Students. Turkish J Sport Med. 2018;53(October):1-8.

20. Weiler R, Chew S, Coombs N, Hamer M, Stamatakis E. Physical activity education in the undergraduate curricula of all UK medical schools. Are tomorrow 's doctors equipped to follow clinical guidelines ? $\mathrm{Br} \mathrm{J}$ Sports Med. 2012;46:1024-6. https://doi.org/10.1136/bjsports-2012-091380

21. Dunlop M, Murray AD. Major limitations in knowledge of physical activity guidelines among UK medical students revealed: implications for the undergraduate medical curriculum. Br J Sports Med. 2013;47:718-20. https://doi.org/10.1136/bjsports-2012-091891

22. Osborne SA, Adams JM, Fawkner S, Kelly P, Murray AD, Oliver CW. Tomorrow's doctors want more teaching and training on physical activity 
for health. Br J Sports Med. 2017;51(8):624-5.

https://doi.org/10.1136/bjsports-2016-096807

23. Wheeler PC, Mitchell R, Ghaly M, Buxton K, Health PP. Primary care knowledge and beliefs about physical activity and health : a survey of primary healthcare team members. Br J Gen Pract. 2017;10:1-10. https://doi.org/10.3399/bjgpopen17X100809

24. Hivert M-F, Arena R, Forman D, Kris-Etherton P, McBride P, Pate R, et al. Medical Training to Achieve Competency in Lifestyle Counseling: An Essential Foundation for Prevention and Treatment of Cardiovascular Diseases and Other Chronic Medical Conditions. Circulation. 2016;134. https://doi.org/10.1161/CIR.0000000000000442

25. Joyner MJ, Sanchis-Gomar F, Lucia A. Exercise medicine education should be expanded. Br J Sports Med. 2017;51(8):625-6. https://doi.org/10.1136/bjsports-2016-096620

26. Valero-Elizondo J, Salami J, Chukwuemeka O, Ogunmoroti O, Blaha MJ, Veledar E, et al. Economic Impact of Moderate-Vigorous Physical Activity Among Those With and Without Established Cardiovascular Disease: 2012 Medical Expenditure Panel Survey. J Am Heart Assoc. 2016;5:111. https://doi.org/10.1161/JAHA.116.003614

Número de citas totales I Total references: 26 (100\%)

Número de citas propias de la revista / Journal's own references: 2 (7,7\%)

Rev.int.med.cienc.act.fís.deporte - vol. 21 - número 83 - ISSN: 1577-0354 